TI 2016-003/III

Tinbergen Institute Discussion Paper

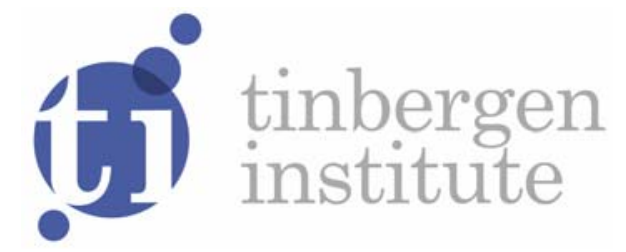

\title{
A Bayesian Approach to Excess Volatility, Short-term Underreaction and Long-term Overreaction during Financial Crises
}

Xu Guo'

Michael McAleer ${ }^{2}$

Wing-Keung Wong 3

Lixing $Z$ hu $^{4}$

' Nanjing University of Aeronautics and Astronautics;

${ }_{2}$ National Tsing Hua University, Taiwan; Erasmus School of Economics, Erasmus University Rotterdam, Tinbergen Institute, the Netherlands; Complutense University of Madrid, Spain;

3 Hong Kong Baptist University, Hong Kong, PR China. 
Tinbergen Institute is the graduate school and research institute in economics of Erasmus University Rotterdam, the University of Amsterdam and VU University Amsterdam.

More TI discussion papers can be downloaded at http://www.tinbergen.nl

Tinbergen Institute has two locations:

Tinbergen Institute Amsterdam

Gustav Mahlerplein 117

1082 MS Amsterdam

The Netherlands

Tel.: +31(0)20525 1600

Tinbergen Institute Rotterdam

Burg. Oudlaan 50

3062 PA Rotterdam

The Netherlands

Tel.: +31(0)10 4088900

Fax: +31(0)10 4089031 


\title{
A Bayesian Approach to Excess Volatility, Short-term Underreaction and Long-term Overreaction during Financial Crises*
}

\author{
Xu Guo \\ College of Economics and Management \\ Nanjing University of Aeronautics and Astronautics
}

\begin{abstract}
Michael McAleer
Department of Quantitative Finance, National Tsing Hua University, Taiwan

Econometric Institute, Erasmus School of Economics,

Erasmus University Rotterdam, Tinbergen Institute, The Netherlands

Department of Quantitative Economics,

Complutense University of Madrid, Spain
\end{abstract}

\author{
Wing-Keung Wong ${ }^{\dagger}$ \\ Department of Economics \\ Hong Kong Baptist University, Hong Kong \\ Lixing Zhu \\ Department of Mathematics \\ Hong Kong Baptist University, Hong Kong
}

January, 2016

${ }^{*}$ The authors are grateful to Markus K. Brunnermeier, Haim Levy, Yew Kwang Ng, Hiroyuki Ozaki, Howard E. Thompson, and Larry Selden for their substantive comments. The third author would also like to thank Robert B. Miller and Howard E. Thompson for their continuous guidance and encouragement. The research is partially supported by grants from Hong Kong Baptist University and Research Grants Council of Hong Kong, Australian Research Council and National Science Council, Taiwan.

$\dagger$ Correspondence: Wing-Keung Wong, Department of Economics, Hong Kong Baptist University, WLB, Shaw Campus, Kowloon Tong, Hong Kong, Tel: (852) 3411-7542, Fax: (852) 3411-5580, email: awong@hkbu.edu.hk 


\begin{abstract}
In this paper, we introduce a new Bayesian approach to explain some market anomalies during financial crises and subsequent recovery. We assume that the earnings shock of an asset follows a random walk model with and without drift to incorporate the impact of financial crises. We further assume the earning shock follows an exponential family distribution to take care of symmetric as well as asymmetric information. By using this model setting, we develop some properties on the expected earnings shock and its volatility, and establish properties of investor behavior on the stock price and its volatility during financial crises and subsequent recovery. Thereafter, we develop properties to explain excess volatility, short-term underreaction, long-term overreaction, and their magnitude effects during financial crises and subsequent recovery.
\end{abstract}

KEYWORDS: Bayesian model; representative and conservative heuristics; excess volatility; underreaction; overreaction; magnitude effects; financial crises. JEL classifications: C11, G01, G11. 


\section{Introduction}

There are several papers that have modeled bubbles and crashes. For example, Abreu and Brunnermeier (2003) use the idea of sequential awareness to develop a theory in which arbitrageurs compete with each other to beat the gun in a stock market. Matsushima (2013) extends their work and shows that rational arbitrageurs are willing to ride the bubble even with incomplete information. He also proves that the bubble can persist for a long period as the unique nash equilibrium outcome. In this paper, we examine the problem of bubbles and crashes by using an alternative perspective, by modeling investor behaviors to ride a bubble during bull and bear markets. The paper will discuss the traditional and behavioral theory for investment.

Explaining market anomalies, such as market excess volatility, overreaction, and underreaction, is one of the most important issues in finance. Classical theorists of market rationality, for example, Fama and French (1996) hypothesize that overreaction and underreaction can be explained by the efficient market paradigm. On the other hand, behaviorial economists such as Barberis, Shleifer, and Vishny (BSV) (1998) combine psychological phenomena with finance theories to explain market anomalies, such as the overreaction and underreaction phenomena.

There are several basic assumptions for the traditional asset-pricing model. Violating the assumptions could result in deviating from the traditional asset-pricing theory in a typical behavioral model. For example, BSV assume that investors adopt conservative and representative heuristics, and the earnings announcements satisfy a random walk following either a trending regime or a mean-reverting regime. This could be used to explain underreaction and overreaction phenomena. In addition, Gervais and Odean (2001) argue that insider traders put higher weights on a security's dividend if they successfully predict their past performance and use the Bayes rule to update their prediction.

It has been observed that investors are too conservative and too slow to vary their prior beliefs when new information arrives. For example, assuming that conservative investors might not pay great attention to the latest earnings announcements, Edwards (1968) develops a Bayesian model that put a lower weight on useful statistical evidence and put greater weight on investors' priors. Nonetheless, Tversky 
and Kahneman (1971), Kahneman and Tversky (1973), and others use the concept of representative heuristics, namely the bias individuals believe population parameters to be "represented" in the latest data, in their experimental studies.

Assuming that people overemphasize the strength of the evidence and de-emphasize its weight when they update their beliefs, Griffin and Tversky (1992) develop a model by combining both conservatism and representativeness. BSV further develop a Bayesian model to explain investors' behavioral biases by using both conservatism and representativeness heuristics in making decisions. Lam, Liu, and Wong (LLW) (2010, 2012) extend their work by introducing a pseudo-Bayesian approach.

In this paper, we modify LLW's pseudo-Bayesian approach by assuming that the earnings shock of an asset follows a random walk model with and without drift to incorporate the impact of financial crises. We also assume earning shocks follow an exponential family distribution to accommodate symmetric as well as asymmetric information. By using this model setting, we establish properties on the expected earnings shock and investor behavior during financial crises and subsequent recovery. Thereafter, we establish new results to explain some market anomalies, including excess volatility, short-term underreaction, long-term overreaction, and their magnitude effects during financial crises and subsequent recovery. For example, the theory developed in this paper infers that excess volatility is proportional to the variance of the earnings shock in the long run, excess volatility increases when the discount rate drops, and conservative (representative) heuristics will decrease (increase) excess volatility.

For short-term underreaction, long-term overreaction, and their magnitude effects, we show that there exist both short-term underreaction and long-term overreaction by using underreaction and/or event approaches. We find that the expected momentum and contrarian profits are positive when the trading period is long enough. In addition, the momentum profit and its magnitude effect are higher in the shorter period, and the contrarian profit and its magnitude effect are higher in the longer period. Moreover, the representative (conservative) heuristic will lead to the contrarian (momentum) profit.

We also show that the representative (conservative) heuristic has to overpower 
the conservative (representative) heuristic to obtain a contrarian (momentum) profit. Furthermore, both momentum and contrarian profits are shown to be sensitive to the discount rate, such that the smaller is the discount rate, the larger are the momentum and contrarian profits. We also find that the expected momentum and contrarian profits are positive when the trading period is long enough. All the above findings hold regardless of the symmetric/asymmetric information on the signs of the earnings shock, and hold during financial crises and during the recovery of the economy. We explain this phenomenon in the Conclusion.

\section{Assumptions and the Model Specification}

\subsection{Earnings Announcements}

The pioneer work of BSV considers a model of market sentiment, in which a representative investor believes that the earnings announcement, $\mathscr{N}_{t}$, of the asset at time $t$ follows a random walk model, such that:

$$
\mathscr{N}_{t}=\mathscr{N}_{t-1}+e_{t}
$$

where $e_{t}$ is an earnings shock at time $t$. Applying the cost-of-capital model (Wong and Chan, 2004), the price, $\mathscr{P}$, of an asset at time $t$ follows:

$$
\begin{aligned}
\mathscr{P}_{t} & =\mathbf{E}_{t}\left[\frac{\mathscr{N}_{t+1}}{1+r}+\frac{\mathscr{N}_{t+2}}{(1+r)^{2}}+\cdots\right] \\
& =\frac{\mathscr{N}_{t}}{r}+\frac{1+r}{r} \times\left[\frac{\mathbf{E}_{t}\left[e_{t+1}\right]}{1+r}+\frac{\mathbf{E}_{t}\left[e_{t+2}\right]}{(1+r)^{2}}+\cdots\right],
\end{aligned}
$$

in which $r$ is the positive discount rate and $\mathbf{E}_{t}[\cdot]$ is the conditional expectation, given the information set $\Omega_{t}$. We assume that $e_{t}$ is $\Omega_{t}$-measurable. Thus, both $\mathscr{P}$ and $\mathscr{N}$ are $\Omega_{t}$-measurable.

In this paper we extend the theory by assuming that the earnings announcement, $\mathscr{N}_{t}$, follows the following random walk model with/without drifts to capture the impact of a financial crises:

$$
\mathscr{N}_{t}= \begin{cases}\delta_{0}+\mathscr{N}_{t-1}+e_{t}, & t_{0} \leq t<t_{1} \\ \delta_{1}+\mathscr{N}_{t-1}+e_{t}, & t_{1} \leq t<t_{2}\end{cases}
$$


in which $\delta_{0}<0$ and $\delta_{1}>0$. In equation (??), we consider an economy with two different states: economic conditions under financial crises and conditions under recovery. We assume that the earnings announcement, $\mathscr{N}_{t}$, follows a random walk model with negative drift, $\delta_{0}$, when financial crises starts at time $t_{0}$, and a random walk with positive drift, $\delta_{1}$, when the economy starts to recover from time $t_{1}$.

BSV assume that the earnings shock is independent and follows a Bernoulli distribution with equal probability on $e_{0}$ or $-e_{0}$. LLW relax this assumption to allow the earnings shock to follow a normal distribution. In this paper, we relax the assumption by assuming that the earnings shock follows an exponential family distribution:

$$
e_{t} \sim f\left(y_{t}\right)=\exp \left\{\left(e_{t} \theta-b(\theta)\right) / a(\phi)+c\left(e_{t} ; \phi\right)\right\}
$$

where $\theta$ is the canonical parameter, $a(>0), b$, and $c$ are known functions, and $\phi$ is the dispersion parameter. The dispersion parameter is assumed to be a constant, either known, or considered as a nuisance parameter.

We note the advantage of using the exponential family distribution is that it is one of the most commonly used continuous distributions, including the normal, gamma, and other distributions, and so, it can fit into situations with symmetric as well as asymmetric distributions. This relaxation is very important because it is well known that market information could be asymmetric. A bear market will be more sensitive to bad news, while a bull market is more sensitive to good news. There are many findings and studies ${ }^{1}$ in the literature supporting the use of the random walk model with drifts for earnings announcement.

One may wonder why investors could foresee when $\left(t_{0}\right)$ a crisis would start and when $\left(t_{1}\right)$ the crisis would end, when the economy would start recovering, and when $\left(t_{2}\right)$ the recovery would end. It is impossible for any investor to know the exact dates of $t_{0}, t_{1}$, and $t_{2}$. However, there are many well-known cycles, for example, the Lunar cycle, January effect, Kitchin cycle, presidential election cycle, Juglar cycle, Kondratieff cycle, and other cycles. ${ }^{2}$ Some smart investors may be able to predict

\footnotetext{
${ }^{1}$ See, for example, Thompson and Wong (1996), Chaudhuri and Wu (2003), Ayers, Li, and Yeung (2011) and the references cited therein.

${ }^{2}$ See, for example, Wong and McAleer (2009) and the references cited therein for more information.
} 
the cycles well, and so they may be able to predict the intervals into which $t_{0}, t_{1}$, and $t_{2}$ fall.

In addition, we note that $t_{0}, t_{1}$, and $t_{2}$ may not be the true dates of the turning points of the cycles. However, if many investors believe that the market will crash at $t_{0}$, they might start selling their stocks and eventually cause the market to crash. This self-fulfilling nature leads to the formation of speculative bubbles (see, for example, Froot, Scharfstein, and Stein (1992)). In addition, Brunnermeier (2001) notes that this episode is an example of herding behavior that is irrational and driven by emotiongreed in the bubbles, fear in financial crashes, and individual investors join the crowd of others in a rush to move in or out of the market.

\subsection{Pseudo-Bayesian Model}

In order to estimate the unknown mean, $\mu$, of the earnings shock, one could use a vague prior $\mathbf{P}_{0}(\mu) \propto 1$ and the likelihood function $\mathbf{L}\left(e_{1}, e_{2}, \cdots, e_{t} \mid \mu\right)=\prod_{i=1}^{t} \mathbf{L}\left(e_{t-i+1} \mid \mu\right)$ in the standard and rational Bayesian approach. This leads to obtain the posterior distribution of $\mu$ conditional on $\left\{e_{1}, e_{2}, \cdots, e_{t}\right\}$, such that $\mathbf{P}\left(\mu \mid e_{1}, e_{2}, \cdots, e_{t}\right) \propto$ $\prod_{i=1}^{t} \mathbf{L}\left(e_{t-i+1} \mid \mu\right){ }^{3}$

Using this standard Bayesian approach will derive the theory to support the traditional efficient market hypothesis and the rational expectation asset-pricing theory (Friedman, 1979). In this situation, any structural irrationally inducing financial anomalies would disappear. However, it is well known that the rational expectations theory is violated empirically (see, for example, Blume and Easley (1982)). In order to circumvent the limitation of the traditional Bayesian model, Slovic (1972) suggests assigning different weights to different observations. If weights $\omega:=\left(\omega_{1}, \omega_{2}, \cdots, \omega_{t}\right)$, LLW propose the following weighted likelihood function:

$$
\mathbf{L}^{\omega}\left(e_{1}, e_{2}, \cdots, e_{t} \mid \mu\right)=\prod_{i=1}^{t} \mathbf{L}\left(e_{t-i+1} \mid \mu\right)^{\omega_{i}} .
$$

\footnotetext{
${ }^{3}$ Readers may refer to Matsumura, Tsui, and Wong (1990) and the references therein cites for more information about the standard and rational Bayesian approaches. We also note that besides using the Bayesian approach, there are alternative approaches that could be used to measure investors' belief; for example, one could use subjective weighting function, as in Levy and Wiener (1998).
} 


\subsection{Weight Assignment}

Assigning general weights on observations enables incorporation of one's prior beliefs into the sample data to examine the price formation process. Brav and Heaton (2002) suggest setting the first half of the weights to one and the second half to zero. LLW suggest using the weighted likelihood function stated in equation (??) and assume investors placing the weight $\omega_{i}$ on the $i^{t h}$ most recent observation, $e_{t-i}$, with $0 \leq \omega_{i} \leq 1$. In this paper, we assume that investors assign the following weights:

$$
0 \leq \omega_{1} \leq \cdots \leq \omega_{m_{0}}=\omega_{m_{0}+1}=\cdots=\omega_{n_{0}}=1 \geq \omega_{n_{0}+1} \geq \cdots \geq 0
$$

for $1 \leq m_{0}<n_{0}$. We note that investors are under the influence of both conservative and representative heuristics if they use weights shown in equation (??), conservatism is obtained when setting $m_{0}>0$, while representativeness is obtained by setting $n_{0}<\infty$. Investors will only have conservative heuristics if $n_{0}=\infty$, and they will only have representative heuristics if $m_{0}=0$. In addition, if $n_{0}=\infty$ and $m_{0}=0$, then all weights will be unity. In this situation, investors will have no behavioral bias. Thus, investors with heuristics shown in equation (??) could represent all other types of investors. Thus, we only study investors with heuristics as shown in equation (??) in this paper.

\section{Basic Properties under the Pseudo-Bayesian Ap- proach}

Under the model discussed in Section ??, we first obtain the following theorem for the stock price and return:

Theorem 1 Applying the pseudo-Bayesian approach with a vague prior, if $N_{t}$ follows the random walk model stated in equation (??) and the likelihood $L^{\omega}(\mu)$ follows the equation stated in equation (??), for any $k \geq 1$, then

$$
\mathbf{E}_{t}\left[e_{t+k}\right]=\frac{\omega_{t} e_{1}+\cdots+\omega_{1} e_{t}}{s_{t}}:=d_{t} \text { and } \sigma_{t}^{2}=\frac{\sigma_{e}^{2} s_{t}}{\sum_{i=1}^{t} \omega_{i}^{2}},
$$

where $s_{t}=\Sigma_{i=1}^{t} \omega_{i}, \mathbf{E}_{t}\left[e_{t+k}\right]$ is the predictive mean of the future earning shock, $e_{t+k}$, and $\sigma_{t}^{2}$ is the posterior variance of $\mu$ given $\left\{e_{1}, e_{2}, \cdots, e_{t}\right\}$. 
We provide a proof of Theorem ?? in the Appendix. LLW develop the results of the rational expectations pricing model in equation (??) when the random walk model is given in equation (??), whereas FLSW extend LLW's work by developing the results of the rational expectations pricing model when the random walk model is given in equation (??). The authors assume that the earnings shock is normally distributed. In this paper, by applying Theorem ?? and assuming the earnings shock, $y_{t}$, follows an exponential family distribution, as stated in equation (??), we extend FLSW's work to relax the normality assumption and obtain the following theorem:

Theorem 2 Under the assumptions stated in Theorem ?? and using the rational expectations pricing model, as stated in equation (??),

a. if $\left\{\mathscr{N}_{t}\right\}$ follows equation (??), then

$$
\mathbf{E}_{t}\left[\frac{\mathscr{P}_{t+k}}{(1+r)^{k}}\right]=\frac{\mathscr{N}_{t}}{r(1+r)^{k}}+\frac{[(1+k) r+1] d_{t}}{r^{2}(1+r)^{k}}, \quad \text { and }
$$

b. if $\left\{\mathscr{N}_{t}\right\}$ follows equation (??), then

$$
\begin{aligned}
& \mathbf{E}_{t}\left[\frac{\mathscr{P}_{t+k}}{(1+r)^{k}}\right] \\
& =\frac{\mathscr{N}_{t}}{r(1+r)^{k}}+\frac{d_{t}[(k+1) r+1]}{r^{2}(1+r)^{k}}+
\end{aligned}
$$

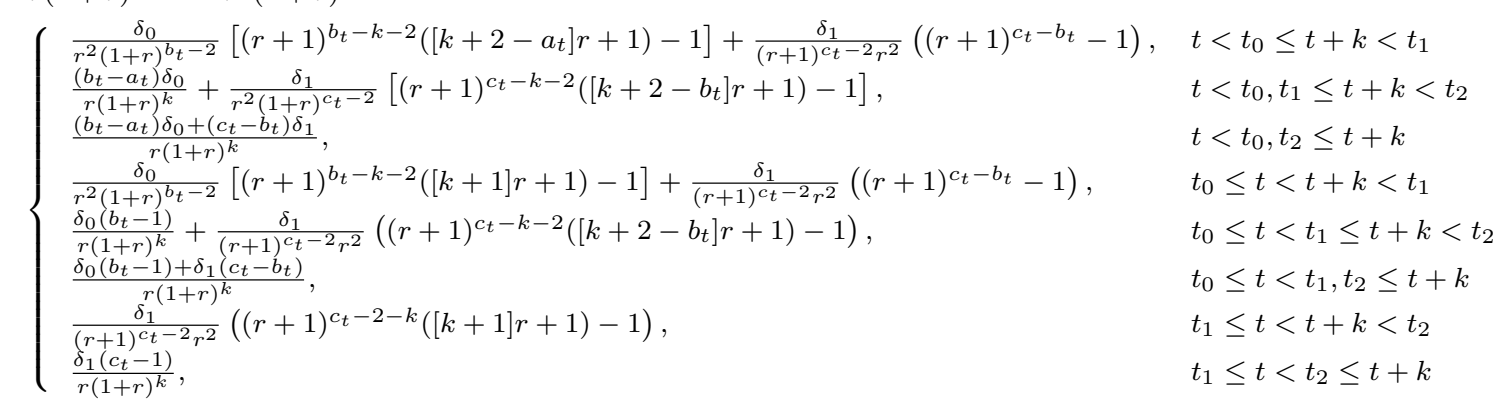

where $\mathscr{P}_{t}$ is the price at time $t, a_{t}=\max \left\{\left\lceil t_{0}-t\right\rceil, 0\right\}, b_{t}=\max \left\{\left\lceil t_{1}-t\right\rceil, 0\right\}$, $c_{t}=\max \left\{\left\lceil t_{2}-t\right\rceil, 0\right\}$, and $d_{t}=\frac{\omega_{t} e_{1}+\cdots+\omega_{1} e_{t}}{s_{t}}$.

From Theorem ??, the current earnings announcement depends on the predictive mean of the future earning shocks, the current earnings announcement, the risk-free interest rate, the duration of the economic recovery or downturn, and the recovery rate of the economy or the deteriorating rate under financial crises. 


\section{Inference on Market Volatility}

According to Theorem ??, whichever proess the random walk $\left\{\mathscr{N}_{t}\right\}$ follows, we have $E_{t}\left[\mathscr{P}_{t+k}\right]=\mathscr{N}_{t} / r+[r(1+k)+1] d_{t} / r^{2}+c_{k}$, where $c_{k}$ may vary, as described in Theorem 2. For $\mathscr{P}_{t}$, we obtain $\mathscr{P}_{t}=\mathscr{N}_{t} / r+(r+1) d_{t} / r^{2}+c_{0}$ and $\mathscr{P}_{t+1}=$ $\mathscr{N}_{t+1} / r+(r+1) d_{t+1} / r^{2}+c_{1}$, where $c_{0}$ and $c_{1}$ are constants, and may differ in value. Consequently, for the 1-period return $\mathscr{R}_{t, t+1}=\mathscr{P}_{t+1}-\mathscr{P}_{t}$ :

$\begin{aligned} \mathscr{R}_{t, t+1}= & \frac{1+r}{r^{2}}\left[\left(\frac{\omega_{t+1}}{s_{t+1}}-\frac{\omega_{t}}{s_{t}}\right) e_{1}+\cdots+\left(\frac{\omega_{2}}{s_{t+1}}-\frac{\omega_{1}}{s_{t}}\right) e_{t}\right]+\left(\frac{1}{r}+\frac{1+r}{r^{2}} \frac{\omega_{1}}{s_{t+1}}\right) e_{t+1} \\ & +c_{1}-c_{0} .\end{aligned}$

From this result, we obtain the following theorem that may be used to draw inferences regarding market volatility:

Theorem 3 Under the assumptions stated in Theorem ??:

a. if $s_{t} \rightarrow \infty$, then market volatility $\operatorname{Var}\left(\mathscr{R}_{t, t+1}\right) \rightarrow \sigma_{e}^{2} / r^{2}$;

b. if there is severe behavioral bias, that is, $s_{t} \rightarrow s_{\infty}<\infty$, then the market volatility $\operatorname{Var}\left(\mathscr{R}_{t, t+1}\right)$ becomes:

$$
\left[\frac{1}{r^{2}}+2 \frac{1}{r} \frac{1+r}{r^{2}} \frac{s_{1}}{s_{\infty}}\right] \sigma_{y}^{2}+\frac{(1+r)^{2}}{r^{4}} \frac{1}{s_{\infty}^{2}} \mathscr{A}_{\infty} \sigma_{e}^{2}
$$

where $\mathscr{A}_{\infty}=\omega_{1}^{2}+\sum_{t=1}^{\infty}\left(\omega_{t+1}-\omega_{t}\right)^{2}$.

In addition, applying Theorem ?? enables us to obtain some interesting observations on excess volatility, as stated in the following property:

Property 4 Under the assumptions stated in Theorem ??, we have:

a. Excess volatility is proportional to the variance of the earnings shock in the long run;

b. If the discount rate and/or investors' anticipated return, $r$, increases, then the excess volatility will be reduced;

c. Conservative (representative) heuristics will decrease (increase) excess volatility; 
d. Parts a to c hold regardless of the symmetric/asymmetric information on the signs of the earnings shock;

e. Parts a to c hold during financial crises and during recovery.

Property ??a could be obtained by applying Part a of Theorem ??. One could obtain Property ??b by conducting simple computations. We provide some remarks for Properties ??c and ??d as follows: If investors adopt a conservative heuristic, then they will choose a positive integer, $m_{0}$, and assign the following weights: $0 \leq$ $\omega_{1}<\cdots<\omega_{m_{0}}=\omega_{m_{0}+1}=\cdots=1$. This leads to $s_{t} \rightarrow \infty$, and excess volatility $\operatorname{Var}\left(\mathscr{R}_{t, t+1}\right) \rightarrow \sigma_{e}^{2} / r^{2}$. On the other hand, if investors select a representative heuristic, they will choose a positive integer, $n_{0}$, and assign weights $1=\omega_{1}=\cdots=\omega_{n_{0}}>$ $\omega_{n_{0}+1}>\cdots \geq 0$. In this situation, if the behavioral biases are severe, then the $\omega_{i}$ are close to 0 for any $i>n_{0}$ and $s_{t} \rightarrow s_{\infty}<\infty$. Thus, the excess volatility will appear in the form of equation (??) and can be larger than that in the conservative heuristic case.

For the third case, investors adopt both conservative and representative heuristics, and so they will choose both $m_{0}$ and $n_{0}$, such that $1 \leq m_{0} \leq n_{0}$, and assign the following weights: $0 \leq \omega_{1}<\cdots<\omega_{m_{0}}=\omega_{m_{0}+1}=\cdots=\omega_{n_{0}}=1>\omega_{n_{0}+1}>\cdots \geq 0$. Their market volatility will be larger than in the conservative heuristic case, but smaller than in the representative heuristic case. If the $\omega_{i}$ are very close to zero for any $i$, with $n_{0}<i$ and $i<m_{0}$, then $s_{t} \rightarrow s_{\infty}<\infty$, and consequently, the excess volatility will also appear in the form of equation (??). In this situation, the excess volatility will be greater than $\sigma_{e}^{2} / r^{2}$. However, when compared with the representative heuristic, as $0 \leq \omega_{1}<1$, the terms $A_{\infty}$ and $s_{1} / s_{\infty}$ will be smaller than those in the representative heuristic case, and so will reduce the excess volatility. We will discuss Properties ??e and $4 \mathrm{f}$ in the Conclusion.

\section{Inferences on Underreaction and Overreaction}

In order to examine the underreaction and overreaction phenomena, we define the lag-one autocovariance, $\gamma_{1}^{k}$, of the $k$-period return as $\gamma_{1}^{k}=\operatorname{Cov}\left(\mathscr{R}_{t, t+k}, \mathscr{R}_{t, t-k}\right)$, where 
$\mathscr{R}_{t, t+k}$ is the $k$-period return and $\mathscr{R}_{t, t-k}$ is the $k$-period return from time $t-k$ to time $t$. The lag-one autocorrelation, $\rho_{1}^{k}$, of the $k$-period return is given as:

$$
\rho_{1}^{k}=\frac{\operatorname{Cov}\left(\mathscr{R}_{t, t+k}, \mathscr{R}_{t, t-k}\right)}{\sqrt{\operatorname{Var}\left(\mathscr{R}_{t, t+k}\right) \operatorname{Var}\left(\mathscr{R}_{t, t-k}\right)}} .
$$

As underreaction (overreaction) is associated with positive (negative) autocorrelation, this leads to the following definition:

Definition 1 An asset displays:

a. a short-term underreaction if $\rho_{1}^{k}>0$ for sufficiently small $k$;

b. a long-term overreaction if $\rho_{1}^{k}<0$ for sufficiently large $k$;

where $\rho_{1}^{k}$ is defined in equation (??).

Based on the above definition, we establish the following theorem:

Theorem 5 Under the assumptions stated in Theorem ??, if investors possess weights as stated in equation (??) and if there is severe behavioral bias, then there exist short-term underreaction and long-term overreaction exhibited as return autocorrelations. That is, there are positive integers $K_{1}$ and $K_{2}$ :

$$
\left\{\begin{array}{l}
\rho_{1}^{k}>0 \quad k \leq K_{1}, \\
\rho_{1}^{k}<0 \quad k>K_{2},
\end{array}\right.
$$

for large $t$, where $\rho_{1}^{k}$ is defined in equation (??). In addition, the limiting correlation coefficients stated in equation (??) are not zero. The results hold regardless of the symmetric/asymmetric information on the signs of the earnings shock, and during financial crises and recovery.

The proof of Theorem ?? is given in the Appendix. We note that it is natural that the results in equation (??) hold for the symmetric as well as asymmetric information on the signs of the earnings shock and during financial crises and recovery. For example, during a crash it is generally common for stock prices to fall day after day. This is exactly what is shown in Theorem ??: that $\rho_{1}^{k}>0$ for $k \leq K_{1}$ for some small integers $K_{1}$; this condition also holds during recovery. On the other hand, during financial crises, one would expect the market to recover after some time; this 
is shown in Theorem ??: that $\rho_{1}^{k}<0$ for $k>K_{2}$ for some large values $K_{2}$. Similarly, during a recovery, one would expect that a recession could arise in the future; this is also shown in Theorem ??: that $\rho_{1}^{k}<0$ for $k>K_{2}$ for some large values $K_{2}$. Similar arguments hold true for the asymmetric information on the signs of the earnings shock. For example, bad news arriving after bad news will result in a further decline in stock price. This is shown in Theorem ?? where $\rho_{1}^{k}>0$ for $k \leq K_{1}$ for small integer $K_{1}$.

We now adopt the event approach used by BSV to describe underreaction and overreaction. It is well known that stock prices can be higher after the company announces good news rather than bad news. However, stock prices can also lower. The market is said to be underreacting in the former situation, and overreacting in the latter situation. We follow the approach used by BSV to quantify such underreaction and overreaction by defining the difference in expected returns after a string of good or bad news, as follows:

$$
\begin{aligned}
\mathscr{U}_{t}(s, j)= & \mathbf{E}\left\{\mathscr{R}_{t+1} \mid e_{t}>\mu+s \sigma_{e}, \cdots, e_{t-j+1}>\mu+s \sigma_{e}\right\} \\
& -\mathbf{E}\left\{\mathscr{R}_{t+1} \mid e_{t}<\mu-s \sigma_{e}, \cdots, e_{t-j+1}<\mu-s \sigma_{e}\right\},
\end{aligned}
$$

where $s$ is the intensity of the content of news and $j$ is the time length of the string of good or bad news. The term $\mathscr{U}_{t}(s, j)\left(-\mathscr{U}_{t}(s, j)\right)$, defined in equation (??) is the expected profit of a momentum (contrarian) trading strategy that dictates buying (selling) when there is a string of good news, and selling (buying) when there is a string of bad news. We can use the sign of $\mathscr{U}_{t}(s, j)$ to measure underreaction and overreaction, as stated in the following definition:

\section{Definition 2}

a. For a sufficiently small $j$, if $\mathscr{U}_{t}(s, j)>0$, then there exists a short-term underreaction in price;

b. for a sufficiently large $j$, if $\mathscr{U}_{t}(s, j)<0$, then there exists a long-term overreaction in price.

Using Definition ??, we establish the following theorem:

Theorem 6 Under the assumptions stated in Theorem ??: 
a. There are short-term underreactions and long-term overreactions in price when an event approach is used. That is, for a given $s>0$ and for large $t$, there exist integers $J_{1}$ and $J_{2}$ such that:

$$
\left\{\begin{array}{l}
\mathscr{U}_{t}(s, j)>0, \quad j \leq J_{1}, \\
\mathscr{U}_{t}(s, j)<0, \quad j \geq J_{2},
\end{array}\right.
$$

where $\mathscr{U}_{t}(s, j)$ is defined in equation (??).

b. When $t \rightarrow \infty$, the expected momentum (contrarian) trading profit, $\mathscr{U}_{t}(s, j)$ $\left(-\mathscr{U}_{t}(s, j)\right)$, is positive for $j \leq J_{1}\left(j \geq J_{2}\right)$.

c. In addition, the results in (a) and (b) above hold regardless of the symmetric/asymmetric information on the signs of the earnings shock.

d. The results in (a) and (b) above hold during financial crises and during recovery.

We provide the proof of Theorem ?? in the Appendix. From Theorems ?? and ?? and their proofs, we obtain the following property for underreaction and overreaction:

Property 7 Under the assumptions stated in Theorem ??, we have:

a. There exist short-term underreactions and long-term overreactions in price when underreaction and/or event approaches are used, and both expected momentum and contrarian profits are positive when the trading period is long enough.

b. The representative (conservative) heuristic contributes to the contrarian (momentum) profit.

c. Overreaction (underreaction) occurs after long (short) periods of good or bad financial performance.

d. The representative (conservative) heuristic has to overpower the conservative (representative) heuristic to obtain a contrarian (momentum) profit to surface.

e. The larger is the discount rate, the smaller are the momentum and contrarian profits, and vice-versa.

f. Observations a-e above hold for asymmetric as well as symmetric information on the signs of the earnings shock, and during financial crises and recovery. 


\section{Inference on the Magnitude Effect}

If the momentum (contrarian) profit $\mathscr{U}_{t}(s, j)\left(-\mathscr{U}_{t}(s, j)\right)$ increases as $s$ or $j$ increases, profits possess a magnitude effect in $s$ or $j$. The following theorems state the magnitude effect for investors using both conservative and representative heuristics.

Theorem $8 \quad$ Under the assumptions stated in Theorem ??, there exists a magnitude effect in $s$ for both long-term overreaction and short-term underreaction that was established in Theorem ??. That is, there exist integers $J_{1}$ and $J_{2}$ such that:

a. The momentum (contrarian) profit $\mathscr{U}_{t}(s, j)\left(-\mathscr{U}_{t}(s, j)\right)$ is positive and strictly increases with $s$ for any sufficiently small (large) $t$ and for any $j<J_{1}\left(j>J_{2}\right)$.

b. The result of the momentum and contrarian profits stated in (a) above hold regardless of the symmetric/asymmetric information on the signs of the earnings shock.

c. The result of the momentum and contrarian profits stated in (a) above hold during financial crises and recovery.

Theorem 9 Under the assumptions stated in Theorem ??:

a. For sufficiently small (large) $j$, the momentum (contrarian) profit based on $j$ consecutive good or bad news increases as $j$ decreases (increases).

b. The result of the momentum and contrarian profits stated in (a) above hold regardless of the symmetric/asymmetric information on the signs of the earnings shock.

c. The result of the momentum and contrarian profits stated in (a) above hold during financial crises and recovery.

We give the proof of Theorem ?? in the Appendix. The proof of Theorem ?? can be easily obtained thereafter. We summarize the results in Theorems ?? and ?? in the following property on the magnitude effect:

Property $10 \quad$ Under the assumptions stated in Theorem ??: 
a. There exist positive momentum and contrarian profits with magnitude effect such that the more does the stock price fluctuates:

$i$. the higher is the momentum profit and its magnitude effect in a short period;

ii. the higher is the contrarian profit and its magnitude effect in a long period.

b. There exist positive contrarian profits with magnitude effect such that:

$i$. the higher is the momentum profit and its magnitude effect if the good/bad news come in the shorter period of time;

ii. the higher is the contrarian profit and its magnitude effect if the good/bad news come in the longer period of time.

c. When the stock price fluctuates more, the momentum profit is positive and monotonic for smaller time periods, while the contrarian profit is positive and monotonic for longer periods.

d. Findings a to $c$ above hold regardless of the symmetric/asymmetric information on the signs of the earnings shock, and hold during financial crises and recovery.

\section{Concluding Remarks}

BSV and others have established Bayesian models to combine psychological phenomena with finance theories to explain market anomalies, such as the overreaction and underreaction phenomena. In this paper, we modified the Bayesian approach to explain market anomalies, including excess volatility, short-term underreaction, long-term overreaction, and their magnitude effects during financial crises and subsequent recovery. It was assumed that: (1) investors exhibit both conservative and representative heuristics that lead them to underweigh recent observations and past observations of the earnings shocks of corporations; (2) the earnings shock of an asset follows a modified random walk model with and without drift, and with an exponential family distribution to incorporate the impact of financial crises; and (3) the 
likelihood function for earning shocks of the stock in a Bayesian paradigm is weighted by investors' behavioral biases.

By using this model setting, we establish properties on the expected earnings shock and it associated volatility. We then established some new results to explain market anomalies, including excess volatility, short-term underreaction, long-term overreaction, and their magnitude effects during financial crises and subsequent recovery.

We noted that the findings in this paper on excess volatility, short-term underreaction, long-term overreaction, and their magnitude effects hold regardless of the symmetric/asymmetric information on the signs of the earnings shock, and hold during financial crises and recovery. One may believe that different market situations and different shapes of the earnings shock should lead to different results. We have illustrated this property for overreaction and underreaction after Theorem ??. We now illustrate this property for excess volatility. One may think that during a market crash, excess volatility would increase sharply. However, Properties ??d and ??e show this is not the case.

We note that this does not mean that different market situations and different shapes of the earnings shock have no effect on excess volatility. These observations only show that the effects of different market situations and different shapes of the earnings shock are already reflected in the use of different heuristics. It does have an effect on excess volatility. For example, when the market is going to crash, investors realize that they could lose most of their investment if they do not sell their stocks. This means that investors will emphasize the recent observations more, and so they select representative heuristics. In this situation, from Property ??c, excess volatility is increasing. Thus, the theory developed from the model could also be used to explain the empirical situation.

The theory developed in this paper could be used to explain investor behaviors in making decisions about financial investments. One may incorporate other information, for example, the economic and financial environment (Fong, Lean, and Wong, 2008), the mean-variance rule (Wong and Ma, 2008; Bai, Hui, Wong, and Zitikis, 2012), CAPM statistics (Leung, Ng and Wong, 2012), VaR rule (Ma and Wong, 
2010), portfolio optimization (Bai, Liu, and Wong, 2009), and portfolio diversification (Egozcue and Wong, 2010) into the theory developed in the paper to make better investment decisions.

There are some other theories that explain the behavior of investors. For example, Levy, Levy, and Solomon $(1994,2000)$ introduce the concepts of rational informed identical (RII) investors. They document that RII investors believe that the stock price may deviate from the fundamental value in the short run but, if it does, it will eventually converge to the fundamental value. On the other hand, the EMBs believe that the stock price accurately reflects the stock's fundamental value.

Wong and Li (1999), Wong (2007), and others extend stochastic dominance theory to obtain some properties for risk averters and risk seekers. Based on the empirical study on momentum profit, Fong, Wong, and Lean (2005) conclude that risk averters prefer to invest in winner portfolios, while Sriboonchitta, Wong, Dhompongsa, and Nguyen (2009) find that risk seekers prefer to invest in loser portfolios. This finding could explain why the momentum profit could still exist after discovery. In addition, Qiao, Clark, and Wong (2012) examine the Taiwan spot and futures markets, and conclude that risk averters prefer to invest in the spot market, whereas risk seekers prefer to invest in the futures market. Hoang, Wong, and Zhu (2015) find that the preference of risk-averse and risk-seeking investors are different in the investment of portfolios with and without gold.

Finally, we note that there are several directions to extend the theory developed in this paper. One area of extension is to develop test statistics for the findings obtained in the paper for different types of investors. Recently, Fabozzi et al. (2013) have introduced statistics to test for the magnitude effect of the overreaction and underreaction hypotheses. Bai et al. (2015) have developed tests to compare the preferences of assets for risk averters and risk seekers. Bai et al. (2011) have developed tests to compare the preferences of assets for investors with S-shaped and reversed Sshaped utility functions. One could incorporate their ideas to develop some statistics to test the findings obtained in the paper for different types of preferences of assets and utility functions. 


\section{Appendix}

Proof of Theorem ??:

Given that:

$$
\mathbf{P}\left(\mu \mid e_{1}, \cdots, e_{t}\right) \propto \prod_{i=1}^{t} \mathbf{L}\left(e_{t-i+1} \mid \mu\right)^{\omega_{i}}=\prod_{i=1}^{t} \exp \left\{\frac{\omega_{i}\left(e_{t-i+1} \theta-b(\theta)\right)}{a(\phi)}+\omega_{i} c\left(e_{t} ; \phi\right)\right\}
$$

the log-likelihood function is:

$$
l(e, \theta)=\sum_{i=1}^{t}\left\{\frac{\omega_{i}\left(e_{t-i+1} \theta-b(\theta)\right)}{a(\phi)}+\omega_{i} c\left(e_{t} ; \phi\right)\right\} .
$$

Consequently, it follows that:

$$
\begin{aligned}
& \frac{\partial l(e, \theta)}{\partial \theta}=\sum_{i=1}^{t}\left\{\frac{\omega_{i}\left(e_{t-i+1}-b^{\prime}(\theta)\right)}{a(\phi)}\right\}, \\
& \frac{\partial^{2} l(e, \theta)}{\partial \theta^{2}}=\sum_{i=1}^{t} \frac{-b^{\prime \prime}(\theta)}{a(\phi)} .
\end{aligned}
$$

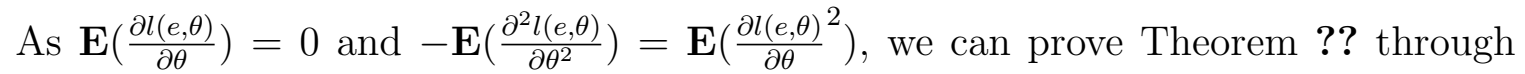
simple calculations.

\section{Proof of Theorem ??:}

Given that:

$$
\begin{aligned}
\mathscr{R}_{t, t+1}= & \frac{1+r}{r^{2}}\left[\left(\frac{\omega_{t+1}}{s_{t+1}}-\frac{\omega_{t}}{s_{t}}\right) e_{1}+\cdots+\left(\frac{\omega_{2}}{s_{t+1}}-\frac{\omega_{1}}{s_{t}}\right) e_{t}\right]+\left(\frac{1}{r}+\frac{1+r}{r^{2}} \frac{\omega_{1}}{s_{t+1}}\right) e_{t+1} \\
& +c_{1}-c_{0},
\end{aligned}
$$

we have:

$$
\begin{aligned}
\operatorname{Var}\left(\mathscr{R}_{t, t+1}\right)= & \left(\frac{1+r}{r^{2}}\right)^{2}\left[\left(\frac{\omega_{t+1}}{s_{t+1}}-\frac{\omega_{t}}{s_{t}}\right)^{2}+\cdots+\left(\frac{\omega_{2}}{s_{t+1}}-\frac{\omega_{1}}{s_{t}}\right)^{2}\right] \sigma_{e}^{2}+\left(\frac{1}{r}+\frac{1+r}{r^{2}} \frac{\omega_{1}}{s_{t+1}}\right)^{2} \sigma_{e}^{2} \\
= & \left(\frac{1}{r^{2}}+\frac{1}{r} \frac{1+r}{r^{2}} \frac{\omega_{1}}{s_{t+1}}\right) \sigma_{e}^{2} \\
& +\left(\frac{1+r}{r^{2}}\right)^{2}\left[\left(\frac{\omega_{1}}{s_{t+1}}\right)^{2}+\left(\frac{\omega_{t+1}}{s_{t+1}}-\frac{\omega_{t}}{s_{t}}\right)^{2}+\cdots+\left(\frac{\omega_{2}}{s_{t+1}}-\frac{\omega_{1}}{s_{t}}\right)^{2}\right] \sigma_{e}^{2} .
\end{aligned}
$$


For the case with $s_{t} \rightarrow \infty$, as $\omega_{i} \leq 1$ for every $i$, we obtain $\frac{\omega_{i}}{s_{t}} \rightarrow 0$. As a result, in this case, $\operatorname{Var}\left(\mathscr{R}_{t, t+1}\right) \rightarrow \frac{1}{r^{2}} \sigma_{e}^{2}$.

When $s_{t} \rightarrow s_{\infty}<\infty$, the result stated in Theorem ?? can be obtained directly from equation (??).

\section{Proof of Theorem ??:}

Applying Theorem ??, we obtain:

$$
\begin{aligned}
& \mathscr{P}_{t+k}=\frac{N_{t+k}}{r}+\frac{(r+1) d_{t+k}}{r^{2}}+c_{k}^{+}, \\
& \mathscr{P}_{t-k}=\frac{N_{t-k}}{r}+\frac{(r+1) d_{t-k}}{r^{2}}+c_{k}^{-},
\end{aligned}
$$

where $c_{k}^{+}$and $c_{k}^{-}$are constants and may differ, as stated in Theorem ??. As a result, we obtain the following for the $k$-period return:

$$
\begin{aligned}
\mathscr{R}_{t, t+k}= & \left(\frac{1}{r}+\frac{(r+1) \omega_{k}}{r^{2} s_{t+k}}\right) e_{t+1}+\cdots+\left(\frac{1}{r}+\frac{(r+1) \omega_{1}}{r^{2} s_{t+k}}\right) e_{t+k} \\
& +\frac{r+1}{r^{2}}\left[\left(\frac{\omega_{t+k}}{s_{t+k}}-\frac{\omega_{t}}{s_{t}}\right) e_{1}+\cdots\left(\frac{\omega_{k+1}}{s_{t+k}}-\frac{\omega_{1}}{s_{t}}\right) e_{t}\right]+c_{k}^{+}-c_{0}, \\
\mathscr{R}_{t, t-k}= & \left(\frac{1}{r}+\frac{(r+1) \omega_{k+2}}{r^{2} s_{t}}\right) y_{t-k+1}+\cdots+\left(\frac{1}{r}+\frac{(r+1) \omega_{1}}{r^{2} s_{t}}\right) e_{t} \\
& +\frac{r+1}{r^{2}}\left[\left(\frac{\omega_{t}}{s_{t}}-\frac{\omega_{t-k}}{s_{t-k}}\right) e_{1}+\cdots\left(\frac{\omega_{k+1}}{s_{t}}-\frac{\omega_{1}}{s_{t-k}}\right) e_{t-k}\right]+c_{0}-c_{k}^{-} .
\end{aligned}
$$

As $e_{i}$ are independent and identically distributed, we obtain:

$$
\begin{aligned}
\operatorname{Cov}\left(\mathscr{R}_{t, t+k}, \mathscr{R}_{t, t-k}\right)= & {\left[\left(\frac{\omega_{t+k}}{s_{t+k}}-\frac{\omega_{t}}{s_{t}}\right)\left(\frac{\omega_{t}}{s_{t}}-\frac{\omega_{t-k}}{s_{t-k}}\right)+\cdots+\left(\frac{\omega_{2 k+1}}{s_{t+k}}-\frac{\omega_{k+1}}{s_{t}}\right)\left(\frac{\omega_{k+1}}{s_{t}}-\frac{\omega_{1}}{s_{t-k}}\right)\right] } \\
& \times\left(\frac{r+1}{r^{2}}\right)^{2} \sigma_{e}^{2} .
\end{aligned}
$$

When the investor has severe behavioral biases, that is $s_{t} \rightarrow s_{\infty}<\infty$, the sign of $\left(\frac{\omega_{2 k+i}}{s_{t+k}}-\frac{\omega_{k+i}}{s_{t}}\right)\left(\frac{\omega_{k+i}}{s_{t}}-\frac{\omega_{i}}{s_{t-k}}\right), i=1, \cdots, t-k$, is determined by the sign of $\left(\omega_{2 k+i}-\right.$ $\left.\omega_{k+i}\right)\left(\omega_{k+i}-\omega_{i}\right)$. If investors possess both conservative and representative heuristics, then they assign weights as $0 \leq \omega_{1}<\cdots<\omega_{m_{0}}=\omega_{m_{0}+1}=\cdots=\omega_{n_{0}}=1>\omega_{n_{0}+1}>$ $\cdots \geq 0$ for $1 \leq m_{0} \leq n_{0}$.

When $k$ is small, the sign of $\left(\omega_{2 k+i}-\omega_{k+i}\right)$ is the same as that of $\left(\omega_{k+i}-\omega_{i}\right)$. For instance, when $i=1,2 k+1<n_{0},\left(\omega_{2 k+1}-\omega_{k+1}\right)>0$, and $\left(\omega_{k+1}-\omega_{1}\right)>0$ hold 
simultaneously. In addition, we have $\left(\omega_{t+k}-\omega_{t}\right)<0$ and $\left(\omega_{t}-\omega_{t-k}\right)<0$. Thus, we obtain $\operatorname{Cov}\left(\mathscr{R}_{t, t+k}, \mathscr{R}_{t, t-k}\right)>0$ for $k<K_{1}$, where $K_{1}$ is a constant.

Similarly, when $k$ is large, the sign of $\left(\omega_{2 k+i}-\omega_{k+i}\right)$ will generally be opposite to that of $\left(\omega_{k+i}-\omega_{i}\right)$. For instance, if $m_{0}<k+1<n_{0}$ and $2 k+1>n_{0}$, then $\left(\omega_{2 k+1}-\omega_{k+1}\right)<0$ and $\left(\omega_{k+1}-\omega_{1}\right)>0$ hold simultaneously. On the other hand, when $t-k>n_{0}$, though $\left(\omega_{t+k}-\omega_{t}\right)<0$ and $\left(\omega_{t}-\omega_{t-k}\right)<0$ still hold, this term is negligible. Recall the assumption that $s_{t} \rightarrow s_{\infty}<\infty$, which infers that $\omega_{t} \rightarrow 0$. Thus, we obtain $\operatorname{Cov}\left(\mathscr{R}_{t, t+k}, \mathscr{R}_{t, t-k}\right)<0$ for $k>K_{2}$, where $K_{2}$ is a constant.

\section{Proof of Theorem ??:}

Let $\mathscr{Z}=\frac{e_{t}-\mu}{\sigma_{e}}, \mathscr{D}_{1}(s)=\mathbf{E}(\mathscr{Z} \mid \mathscr{Z}>s)$, and $\mathscr{D}_{2}(s)=\mathbf{E}(\mathscr{Z} \mid \mathscr{Z}<-s)$. Applying Lemma 1 in LLW, we have:

$$
\begin{gathered}
\mathbf{E}\left[\mathscr{R}_{t+1} \mid e_{t}>\mu+s \sigma_{e}, \cdots, e_{t-j+1}>\mu+s \sigma_{e}\right]=\frac{1+r}{r^{2}} \sigma_{e}\left[\frac{\mathscr{K}_{t}}{s_{t} s_{t+1}}+\Delta(t, j) \mathscr{D}_{1}(s)\right]+c_{1}-c_{0}, \\
\mathbf{E}\left[\mathscr{R}_{t+1} \mid e_{t}<\mu-s \sigma_{e}, \cdots, e_{t-j+1}<\mu-s \sigma_{e}\right]=\frac{1+r}{r^{2}} \sigma_{e}\left[\frac{\mathscr{K}_{t}}{s_{t} s_{t+1}}+\Delta(t, j) \mathscr{D}_{2}(s)\right]+c_{1}-c_{0},
\end{gathered}
$$

where $\mathscr{K}_{t}=\frac{\mu}{\sigma_{e}}\left[\frac{r}{r+1}\right] s_{t} s_{t+1}$ and $\Delta(t, j)=\frac{s_{j+1}-s_{1}}{s_{t+1}}-\frac{s_{j}}{s_{t}}$. Consequently, we obtain:

$$
\mathscr{U}_{t}(s, j)=\frac{1+r}{r^{2}} \sigma_{e} \Delta(t, j)\left(\mathscr{D}_{1}(s)-\mathscr{D}_{2}(s)\right) .
$$

Obviously, $\mathscr{D}_{1}(s)>0$ and $\mathscr{D}_{2}(s)<0$. As a result, the sign of $\mathscr{U}_{t}(s, j)$ depends on the sign of $\Delta(t, j)=\frac{s_{j+1}-s_{1}}{s_{t+1}}-\frac{s_{j}}{s_{t}}$. As $s_{t} \rightarrow s_{\infty}<\infty$, when $t$ is sufficiently large, the sign of $\Delta(t, j)$ is determined by the sign of $s_{j+1}-s_{1}-s_{j}=\omega_{j}-\omega_{1}$. When $j$ is very small, the conservative heuristic guarantees that $\omega_{1}<\omega_{j}$, so that $\mathscr{U}_{t}(s, j)>0$ for $j<J_{1}$. On the other hand, when $j$ is large enough, $\omega_{j}$ can be arbitrarily small because of the assumption that $s_{t} \rightarrow s_{\infty}<\infty$. Consequently, we have $\omega_{1}>\omega_{j}$, so that $\mathscr{U}_{t}(s, j)<0$ for $j>J_{2}$.

\section{Proof of Theorem ??:}

Let $h(s)=\mu+s \sigma_{e}$ and $g(s)=\mu-s \sigma_{e}$, so that:

$$
\begin{aligned}
& \mathscr{D}_{1}(s)=\frac{\int_{h(s)}^{\infty} y f(y) d y}{\int_{h(s)}^{\infty} f(y) d y \cdot \sigma_{e}}-\frac{\mu}{\sigma_{e}}, \\
& \mathscr{D}_{2}(s)=\frac{\int_{0}^{g(s)} y f(y) d y}{\int_{0}^{g(s)} f(y) d y \cdot \sigma_{e}}-\frac{\mu}{\sigma_{e}} .
\end{aligned}
$$


This leads to

$$
\begin{aligned}
\mathscr{D}_{1}^{\prime}(s) & =\frac{f(h(s)) h^{\prime}(s)\left[\int_{h(s)}^{\infty} y f(y) d y-h(s) \int_{h(s)}^{\infty} f(y) d y\right]}{\left(\int_{h(s)}^{\infty} f(y) d y\right)^{2} \cdot \sigma_{e}} \\
& =\frac{f(h(s)) h^{\prime}(s) \int_{h(s)}^{\infty} f(y) d y(\xi-h(s))}{\left(\int_{h(s)}^{\infty} f(y) d y\right)^{2} \cdot \sigma_{e}},
\end{aligned}
$$

where $h(s)<\xi<\infty$, and the last equation follows from applying the mean value theorem of integrals. Thus, we have $\mathscr{D}_{1}^{\prime}(s)>0$.

By using a similar argument, we obtain:

$$
\begin{aligned}
\mathscr{D}_{2}^{\prime}(s) & =\frac{f(g(s)) g^{\prime}(s)\left[g(s) \int_{0}^{g(s)} f(y) d y-\int_{0}^{g(s)} y f(y) d y\right]}{\left(\int_{0}^{g(s)} f(y) d y\right)^{2} \cdot \sigma_{e}} \\
& =\frac{f(g(s)) g^{\prime}(s) \int_{0}^{g(s)} f(y) d y(g(s)-\zeta)}{\left(\int_{0}^{g(s)} f(y) d y\right)^{2} \cdot \sigma_{e}},
\end{aligned}
$$

where $0<\zeta<g(s)$. We note that $g^{\prime}(s)=-\sigma_{e}<0$, so that $\mathscr{D}_{2}^{\prime}(s)<0$. Consequently, based on this result, and by using an argument similar to that in Theorem ??, Theorem ?? is obtained. 


\section{References}

Abreu, D. and M. Brunnermeier, 2003. Bubbles and crashes, Econometrica 71, 173204.

Ayers, B. C., Li, O. Z., Yeung, P. E., 2011. Investor trading and the post-earningsannouncement drift, Accounting Review 86(2), 385-416.

Bai, Z.D., Hui, Y. C., Wong, W.K., Zitikis, R., 2012. Evaluating prospect performance: Making a case for a non-asymptotic UMPU test, Journal of Financial Econometrics 10(4), 703-732.

Bai, Z.D., Li, H., Liu, H.X., Wong, W.K., 2011. Test statistics for prospect and Markowitz stochastic dominances with applications, Econometrics Journal 14(2), 278-303.

Bai, Z.D., Li, H., McAleer, M., Wong, W.K., 2015. Stochastic dominance statistics for risk averters and risk seekers: An analysis of stock preferences for USA and China, Quantitative Finance 15(5), 889-900.

Bai, Z.D., Liu, H.X., Wong, W.K., 2009. Enhancement of the applicability of Markowitz's portfolio optimization by utilizing random matrix theory, Mathematical Finance 19(4), 639-667.

Barberis, N., Shleifer, A., Vishny, R., 1998. A model of investor sentiment, Journal of Financial Economics 49, 307-343.

Blume, L.E., Easley, D., 1982. Learning to be rational, Journal of Economic Theory $26,340-351$.

Brav, A., Heaton, J.B., 2002. Competing theories of financial anomalies. Review of Financial Studies 15, 575-606.

Brunnermeier, M. K., 2001. Asset pricing under asymmetric information: Bubbles, crashes, technical analysis, and herding, Oxford University Press.

Chaudhuri, K., Wu, Y., 2003. Random walk versus breaking trend in stock prices: Evidence from emerging markets. Journal of Banking \& Finance 27, 575-592.

Edwards, W., 1968. Conservatism in human information processing, in Formal representation of human judgment, Kleinmutz, B. (ed.), New York: Wiley.

Egozcue, M., Wong, W.K., 2010. Gains from diversification on convex combinations: A majorization and stochastic dominance approach. European Journal of Operational Research 200, 893-900. 
Fabozzi, F.J., Fung, C.Y., Lam, K., Wong, W.K., 2013, Market overreaction and underreaction: Tests of the directional and magnitude effects, Applied Financial Economics 23(18), 1469-1482.

Fama, E.F., French, K.R., 1996. Multifactor explanations of asset pricing anomalies. Journal of Finance 51(1), 55-84.

Fong, W.M., Lean, H.H., Wong, W.K., 2008. Stochastic dominance and behavior towards risk: The market for internet stocks. Journal of Economic Behavior and Organization 68(1), 194-208.

Fong, W.M., Wong, W.K., Lean, H.H., 2005. International momentum strategies: A stochastic dominance approach, Journal of Financial Markets 8, 89-109.

Friedman, B.M., 1979. Optimal expectations and the extreme information assumptions of rational expectations' macromodels. Journal of Monetary Economics 5, 23-41.

Froot, K.A., Scharfstein, D.S., Stein, J.C. 1992. Heard on the street : Information inefficiencies in a market with short-term speculators. Journal of Finance 47, 1461-1484.

Gervais, S., Odean, T., 2001. Learning to be overconfident. Review of Financial Studies 14(1), 1-27.

Griffin, D., Tversky, A., 1992. The weighing of evidence and the determinants of confidence. Cognitive Psychology 24, 411-435.

Hoang, T.H.V., Wong, W.K., Zhu, Z.Z., 2015. Is gold different for risk-averse and risk-seeking investors? An empirical analysis of the Shanghai Gold Exchange, Economic Modelling 50, 200-211.

Kahneman, D., Tversky, A., 1973. On the psychology of prediction. Psychological Review 80, 237-251.

Lam, K., Liu, T., Wong, W.K., 2010. A pseudo-Bayesian model in financial decision making with implications to market volatility, under- and overreaction, European Journal of Operational Research 203(1), 166-175.

Lam, K., Liu, T., Wong, W.K., 2012. A new pseudo Bayesian model with implications to financial anomalies and investors' behaviors. Journal of Behavioral Finance 13(2), 93-107.

Levy, H., Levy, M., Solomon, S., 1994. A microscopic model of the stock market: Cycles, booms, and crashes. Economics Letters 45(1), $103 ? 11$. 
Levy, H., Levy, M., Solomon, S., 2000. The microscopic simulation of financial markets: from investor behavior to market phenomena, Academic Press.

Levy, H., Wiener, Z., 1998. Stochastic dominance and prospect dominance with subjective weighting function. Journal of Risk and Uncertainty 16(2), 147-163.

Ma, C., Wong, W.K., 2010. Stochastic dominance and risk measure: A decisiontheoretic foundation for VaR and C-VaR. European Journal of Operational Research 207, 927-935.

Matsumura, E.M., Tsui, K.W., Wong, W.K., 1990. An extended multinomial-Dirichlet model for error bounds for dollar-unit sampling. Contemporary Accounting Research 6(2-I), 485-500.

Matsushima, H., 2013. Behavioral aspects of arbitrageurs in timing games of bubbles and crashes. Journal of Economic Theory 148(2), 858-870.

Qiao Z., Clark, E., Wong, W. K., 2012. Investors' preference towards risk: Evidence from the Taiwan stock and stock index futures markets. Accounting \& Finance, DOI: 10.1111/j.1467-629X.2012.00508.x.

Slovic, P., 1972. Psychological study of human judgment: Implications for investment decision making. Journal of Finance 27, 779-799.

Sriboonchitta, S., Wong, W.K., Dhompongsa, D., Nguyen, H.T., 2009. Stochastic Dominance and Applications to Finance, Risk and Economics. Chapman and Hall/CRC, Boca Raton, Florida.

Thompson, H.E., Wong, W.K., 1996. Revisiting 'dividend yield plus growth' and its applicability. Engineering Economist 41(2), 123-147.

Tversky, A., Kahneman, D., 1971. Belief in the law of small numbers. Psychological Bulletin 76, 105-110.

Wong, W.K., 2007. Stochastic dominance and mean-variance measures of profit and loss for business planning and investment. European Journal of Operational Research 182, 829-843.

Wong, W.K., Chan, R., 2004. The estimation of the cost of capital and its reliability. Quantitative Finance 4(3), 365-372.

Wong, W.K., Li, C.K., 1999. A note on convex stochastic dominance. Economics Letters 62, 293-300.

Wong, W.K., Ma, C., 2008. Preferences over location-scale family. Economic Theory 37(1), 119-146. 
Wong, W.K., McAleer, M., 2009. Mapping the Presidential election cycle in US stock markets. Mathematics and Computers in Simulation 79(11), 3267-3277. 